\title{
Gliadin activates mucosal cell mediated immunity in cultured rectal mucosa from coeliac patients and a subset of their siblings
}

\author{
R Troncone, G Mazzarella, N Leone, M Mayer, M De Vincenzi, L Greco, S Auricchio
}

\begin{abstract}
Background-CD3 and $\gamma \delta$ cells in the rectal mucosa increase after local instillation of gluten in children with coeliac disease and in half of their siblings.

Aim-To establish an in vitro system for assessing immunological changes induced by gluten in the rectum.

Patients and Methods-Rectal biopsy specimens obtained from 13 treated coeliac children, nine of their siblings, and nine controls were cultured in vitro with a peptic-tryptic digest of gliadin or ovalbumin. CD3 and CD25 cells were counted, and the expression of adhesion molecules evaluated.

Results-In the lamina propria of coeliac biopsy samples cultured with gliadin, but not in those from controls, the expression of vascular cell adhesion molecule 1 (VCAM-1) was enhanced, and the number of $\mathrm{CD} 25$ cells was significantly higher than in those cultured in medium alone; the density of intraepithelial CD3 cells was also significantly higher. No differences were noted in coeliac biopsy specimens cultured with ovalbumin. A discriminant analysis allowed correct classification of all controls and all coeliacs but one, but three of nine siblings were allocated to the coeliac group.

Conclusions-Our data confirm that gliadin is able to activate cell mediated immunity in the rectal mucosa in coeliac patients and in a subset of their first degree relatives.

(Gut 1998;43:484-489)
\end{abstract}

Keywords: coeliac disease; gliadin; rectum; organ culture; mucosal immunity

Istituto di Scienze dell'Alimentazione CNR, Avellino, Italy G Mazzarella

N Leone

Laboratory for Metabolism and Pathological Biochemistry, Istituto Superiore di Sanita', Rome, Italy

M De Vincenzi

Correspondence to: Dr R Troncone,

Dipartimento di Pediatria, via Sergio Pansini 5, I-80131 Napoli, Italy.

Accepted for publication 31 March 1998

mechanisms by which wheat gliadins prolamins from other toxic cereals (rye, barley, and oats) produce lesions to the small intestine of patients with coeliac disease are not completely understood, although most investigators favour the activation of intestinal $\mathrm{T}$ lymphocytes by gliadin as the underlying abnormality (reviewed by Marsh $^{1}$ and Troncone et $a l^{2}$ ). However, jejunum is not the only site of the gastrointestinal tract involved in coeliac disease. In recent years, the use of immunohistochemistry and computerised image analysis in cell quantification has definitively shown the presence of gluten dependent inflammatory changes in the rectal mucosa. Increased numbers of $\mathrm{CD} 3$ and $\gamma \delta$ lymphocytes, plasma cells, mast cells and mononuclear cells expressing interleukin 2 receptors $(\mathrm{CD} 25)^{3}$ and increased expression of vascular cell adhesion molecule 1 (VCAM-1) ${ }^{4}$ have all been shown in the rectal mucosa of untreated coeliac patients; these changes can all be reversed, with the sole exception of the increase in the number of mast cells, by dietary treatment. ${ }^{34}$ These data suggest that, like the jejunum, the rectal mucosa in untreated coeliac disease is a site of gluten dependent activated mucosal cell mediated immunity. As the rectum provides easy access for obtaining multiple mucosal samples, the response of rectal mucosa to local gluten challenge was investigated, as it may help us both to understand the phenomena triggered by mucosal contact with the antigen gliadin and to diagnose coeliac disease. Significant changes in lamina propria volume and numbers of mast cells, neutrophils, and lymphocytes have been shown in the rectal mucosa in coeliac disease after challenge with a peptictryptic digest of gluten. ${ }^{5}$ We have recently reappraised rectal gluten challenge in a paediatric setting: a substantial infiltration of lymphocytes was noted in the epithelium six hours after a gliadin enema. These data were paralleled by immunohistochemical analysis showing recruitment of CD 3 and $\gamma \delta$ lymphocytes. ${ }^{6}$ Interestingly, about half of the siblings of children with coeliac disease also reacted to rectal instillation of gliadin. ${ }^{6}$

Most of our information on the immunological consequences of gluten challenge at the mucosal level have been obtained in vitro using organ culture of jejunal biopsy samples. ${ }^{7}$ In this study, taking advantage of the long experience gained in our laboratory in organ culture, we have extended previous data from in vivo rectal challenge studies, and established an in vitro system, with the aim to be able to assess fully immunological changes induced by gluten in the rectal mucosa.

\section{Subjects and methods}

PATIENTS

Thirteen coeliac patients (five boys and eight girls, median age 11 years, range 8-19) were studied. All cases were diagnosed according to the criteria laid down in 1971 by the European Society for Paediatric Gastroenterology and Nutrition (ESPGAN) ${ }^{8}$; all had shown subtotal villous atrophy while on a gluten containing 
Table 1 Monoclonal antibodies used in this study

\begin{tabular}{lllll}
\hline Designation & Specificity & Isotype & Working dilution & Source \\
\hline T3-4B5 & CD3 & IgG1 & $1: 200$ & Dako \\
ACT-1 & CD25 & IgG1 & $1: 25$ & Dako \\
3C5 & ICAM-1 & IgG1 & $1: 500$ & Genzyme \\
4B9 & VCAM-1 & IgG1 & $1: 500$ & Genzyme \\
BB-11 & ELAM-1 & IgG1 & $1: 500$ & Genzyme \\
\hline
\end{tabular}

diet, a good clinical and histological response to a gluten-free diet, and histological relapse after gluten challenge. At the time of the study all had been on a gluten-free diet for at least two years. Nine disease controls (six boys and three girls, median age five years, range 2-10) were also studied, for whom the final diagnosis was different from coeliac disease (four were affected by constipation, two by recurrent abdominal pain, two by failure to thrive, and one by intolerance to cow's milk protein). Finally, nine healthy siblings of coeliac patients (seven boys and two girls, median age 12 years, range 6-20) were investigated. Siblings were also challenged in vivo with $2 \mathrm{~g}$ Frazer's peptictryptic digest of gliadin (Sigma Chemical Company, St Louis, Missouri, USA). The protein was dissolved in $20 \mathrm{ml}$ sterile water and instilled into the lower rectum via a small catheter. A biopsy specimen was obtained six hours after the challenge using a Crosby capsule positioned $10 \mathrm{~cm}$ from the anal verge.

Informed consent was obtained from all patients and a parent/guardian when appropriate. The study design was approved by the university ethics committee.

PROCESSING OF RECTAL BIOPSY SPECIMENS AND ORGAN CULTURE

One or two rectal biopsy specimens were obtained using a Crosby capsule positioned 10 $\mathrm{cm}$ from the anal verge. Immediately after excision, each specimen was washed in 0.15 $\mathrm{mol} / \mathrm{l} \mathrm{NaCl}$ and sliced into two or three approximately equal sized pieces. One piece was oriented and embedded in OCT compound (Tissue Tek; Miles Laboratories, Elkhart, Indiana, USA), snap frozen in isopentane cooled in liquid nitrogen, and stored at $-70^{\circ} \mathrm{C}$ until cryosectioning. The remaining pieces were placed on a stainless steel mesh positioned over the central well of an organ culture dish (Falcon) with the mucosal surface of the specimen uppermost. The well was then filled with culture medium so as just to reach the cut surface of the specimen $(10 \mathrm{ml}$ culture medium consisted of Trowell's T8 medium $(6.5 \mathrm{ml})$, NCTC 135 medium $(2.5 \mathrm{ml})$, fetal calf serum $(1 \mathrm{ml})$, supplemented with 100 $\mathrm{U} / \mathrm{ml}$ penicillin, $100 \mathrm{mg} / \mathrm{ml}$ streptomycin, and $5 \mu \mathrm{g} / \mathrm{ml}$ gentamicin). The dishes were placed in a sterile anaerobic jar which was gassed with $95 \%$ oxygen $/ 5 \%$ carbon dioxide and incubated at $37^{\circ} \mathrm{C}$. Biopsy fragments were cultured in the presence of medium alone or a gliadin peptictryptic digest at a concentration of $1 \mathrm{mg} / \mathrm{ml}$. The digest was prepared as previously described. ${ }^{9}$ In some experiments the biopsy specimens were also cultured in the presence of ovalbumin (Sigma) at the same final concentration. After 24 hours of culture, the tissue was embedded in OCT compound, snap frozen in liquid nitrogen, and prepared for cryosectioning as described above.

STAINING TECHNIQUE

Cryostat sections $(5 \mu \mathrm{m})$ were air dried at room temperature and then fixed in acetone for 10 minutes. All the sections were repeatedly washed at room temperature in Tris buffered saline (TBS), then incubated in normal rabbit serum (1:100 in TBS for 30 minutes), and stained by the peroxidase-antiperoxidase (PAP) or alkaline phosphatase/anti-alkaline phosphatase (APAAP) method. The sections were individually tested with the monoclonal antibodies (table 1). After one hour's incubation at room temperature, the sections were incubated for 30 minutes with rabbit antimouse serum (Dako, Copenhagen, Denmark), and for a further 30 minutes with APAAP complex to stain CD25 cells and adhesion molecules or with PAP complex (Dako) to stain CD3 cells. Slides were washed in TBS for 10 minutes after each antibody incubation. The reaction product was developed by a final incubation of five minutes with 2-amino-9ethylcarbazole when the PAP complex was used; alternatively, when the APAAP complex was used, the immune reaction product was developed by stirring continuously for three minutes with solution containing $20 \mathrm{mg}$ naphthol-AS-biphosphate in $0.5 \mathrm{ml} \mathrm{N}, N-$ dimethylformamide added to $0.2 \mathrm{ml}$ sodium nitrite (Sigma), $0.08 \mathrm{ml}$ of New Fucsin (Merck, Sharp and Dohme, West Point, Philadelphia, USA), $40 \mathrm{ml}$ TBS, $\mathrm{pH} 8.7$, and 17.5 $\mathrm{mg}$ levamisole (Sigma). Omission of the primary antibody served as a control against non-specific antibody binding. The sections were finally stained with Mayer's haematoxylin and mounted. Non-immune mouse immunoglobulins of the same isotype were used as primary antibodies for control of specificity.

MORPHOMETRIC ANALYSIS

At least four slides for each sample were evaluated at $40 \times$ magnification. The density of CD3 cells in the surface epithelium was determined by counting the number of stained cells as a percentage of 100 enterocytes; at least 500 enterocytes were counted in each sample. The number of CD25 cells was evaluated within a total area of $1 \mathrm{~mm}^{2}$ of lamina propria, using a microscope with a calibrated ocular graticule aligned parallel to the muscularis mucosae. In five experiments all measurements were repeated by two independent observers (GM and NL). The coefficient of variation - that is, the mean absolute difference between observers multiplied by 100 and divided by the mean pooled value) was $15 \%$ for CD25 cells and $8 \%$ for CD3 cells. The expression of adhesion molecules was evaluated in terms of staining intensity and graded on an arbitrary scale of no staining $=0$, weak staining $=1$, moderate staining $=2$, strong staining $=3$. The slides were independently analysed in a blinded manner by two observers. 


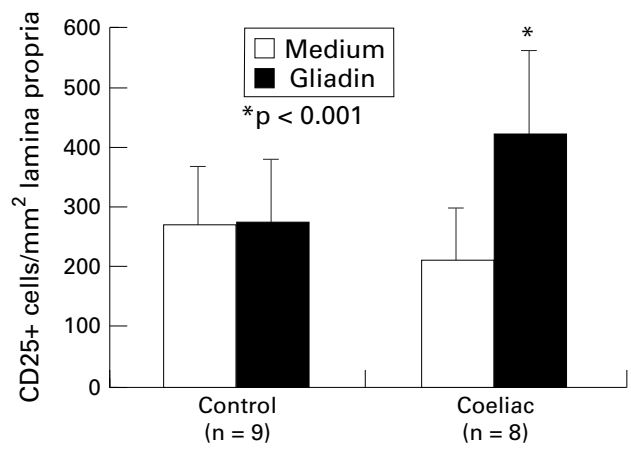

Figure 1 CD25 mononuclear cells in the lamina propria of rectal biopsy specimens cultured in vitro with gliadin. Bars represent means (SD) of paired samples cultured with medium alone and with gliadin.

STATISTICAL ANALYSIS

Data were edited, corrected, and assessed by descriptive analysis from the Statistical Package for Social Sciences. Student's two tailed $t$ test for paired samples was used to compare specimens cultured with gliadin with those exposed to medium alone for the same incubation period. Non-parametric tests (Wilcoxon two tailed) were also applied, and the results
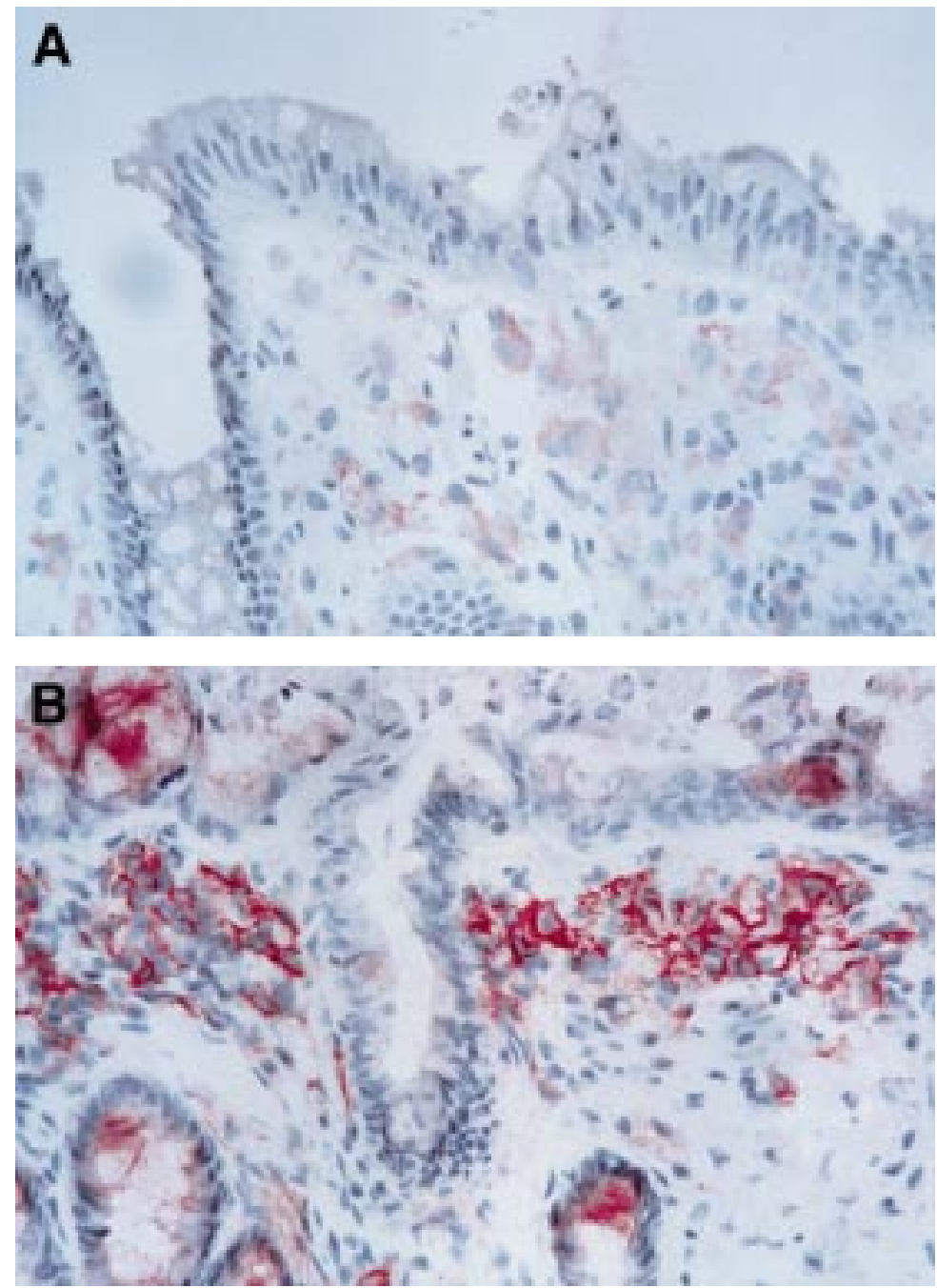

Figure 2 Mononuclear cells expressing interleukin 2 receptor (CD25) in the rectal mucosa from a coeliac patient cultured in vitro with medium only $(A)$ or with a peptic-tryptic digest of gliadin (B). In the latter, increased numbers of immunostained cells are evident particularly in the subepithelial region. Original magnification $\times 400$.

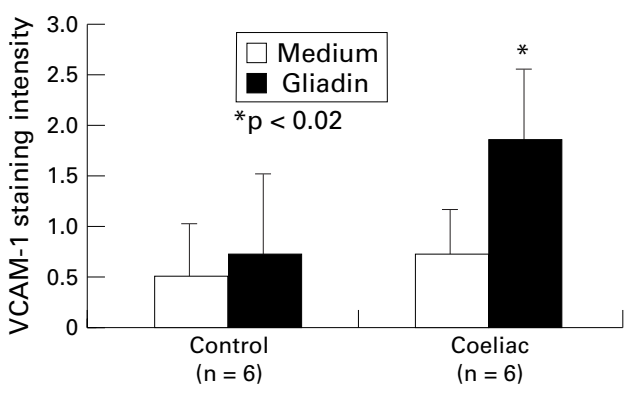

Figure 3 Vascular cell adhesion molecule 1 (VCAM-1) expression in the lamina propria of rectal biopsy specimens cultured in vitro with gliadin. Expression of adhesion molecules was evaluated in terms of staining intensity and graded on an arbitrary scale of no staining $=0$, weak staining $=1$, moderate staining $=2$, strong staining $=3$. Bars represent means (SD) of paired samples cultured with medium alone and with gliadin.

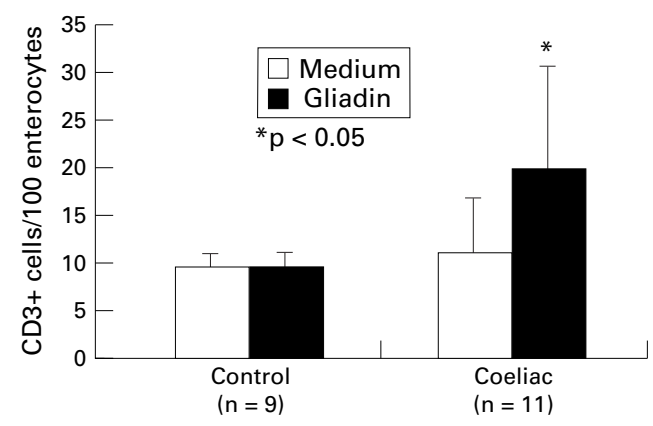

Figure 4 CD3 intraepithelial lymphocytes in rectal biopsy specimens cultured in vitro with gliadin. Bars represent means (SD) of paired samples cultured with medium alone and with gliadin.

are concordant with those obtained using parametric tests. Multivariate analysis was performed by a stepwise discriminant function. Only variables contributing with $\mathrm{p}<0.001$ to the discrimination of groups were included.

\section{Results}

ORGAN CULTURE OF RECTAL MUCOSA FROM COELIAC PATIENTS IN THE PRESENCE OF GLIADIN In the lamina propria of coeliac rectal biopsy specimens cultured in the presence of medium alone, the number of cells expressing the receptor for interleukin 2 (CD25 cells) (mean (SD) $206(88) / \mathrm{mm}^{2}$ ) was significantly ( $<<0.01$; paired $t$ test) higher than in non-cultured specimens (31 (24)). However, the number of CD25 cells in specimens cultured in the presence of a peptic-tryptic digest of gliadin $(413(142))$ was significantly $(\mathrm{p}<0.001)$ higher than in those cultured in medium alone (fig 1 ). Both $\mathrm{T}$ cells and macrophages were present in this population. The presence of large cell aggregates, particularly in the subepithelial region (fig 2), rendered the differential counting of macrophages and $\mathrm{T}$ cells difficult. Moreover, it is difficult to conclude whether most CD25 cells are resident subepithelial macrophages or whether they are from a deeper layer.

Expression of adhesion molecules was also evaluated in six cultures with gliadin from controls and six from coeliac patients to look for other evidence of activated mucosal cell mediated immunity. Intercellular adhesion molecule 1 (ICAM-1) was expressed strongly on all cells 
Table 2 Discrimination function parameters used to compute the discriminant score. Discriminant scores are obtained by multiplying the observed value by the standardised discriminant function coefficients and adding the constant

\begin{tabular}{ll}
\hline Variables that contribute to the discrimination & $\begin{array}{l}\text { Discriminant function coefficients } \\
\text { (unstandardised) }\end{array}$ \\
\hline Number of lamina propria CD25 & 0.011 \\
Percentage increase of epithelium CD3 & 0.006 \\
Constant & -2.158 \\
\hline
\end{tabular}

and vessels; endothelial-leucocyte adherence molecule 1 (ELAM-1) was present in moderate amounts on all venules and in small amounts on a few leucocytes, and vascular cell adhesion molecule 1 (VCAM-1) was expressed in moderate amounts on mononuclear cells and only weakly or not at all on blood vessels. However, only VCAM-1 expression was significantly $(\mathrm{p}<0.02)$ enhanced, particularly on mononuclear cells of biopsy specimens cultured for 24 hours with gliadin, as compared with those cultured with medium alone (fig 3 ).

Finally, a significant $(\mathrm{p}<0.05)$ increase in CD3 intraepithelial lymphocytes was seen, again in coeliac biopsy samples cultured with

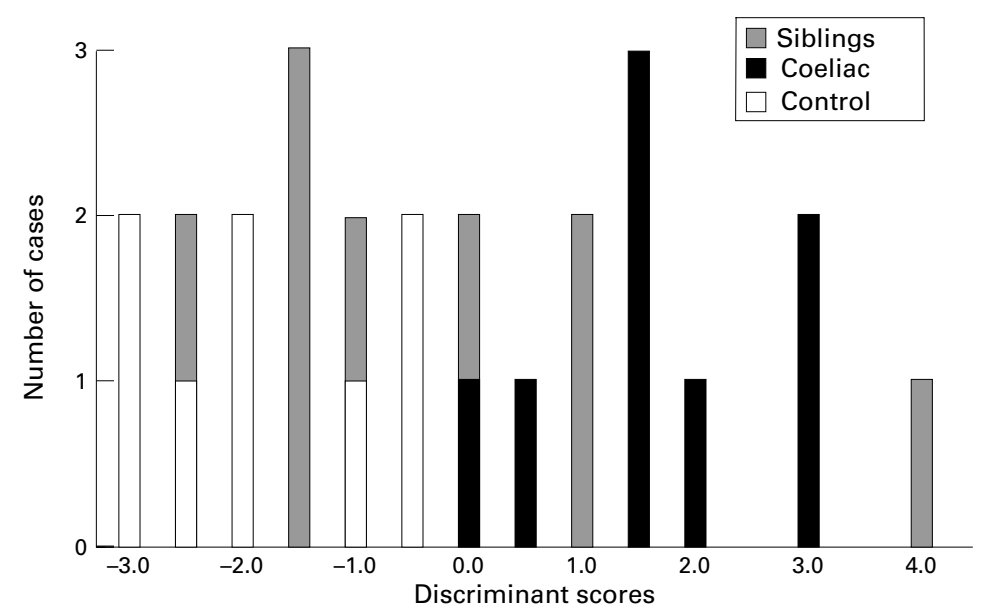

Figure 5 Discriminant analysis of immunohistochemical data: distribution of discriminant scores in controls, patients with coeliac disease, and siblings of patients with coeliac disease. Discriminant scores were computed using the discrimination function parameters reported in table 2.
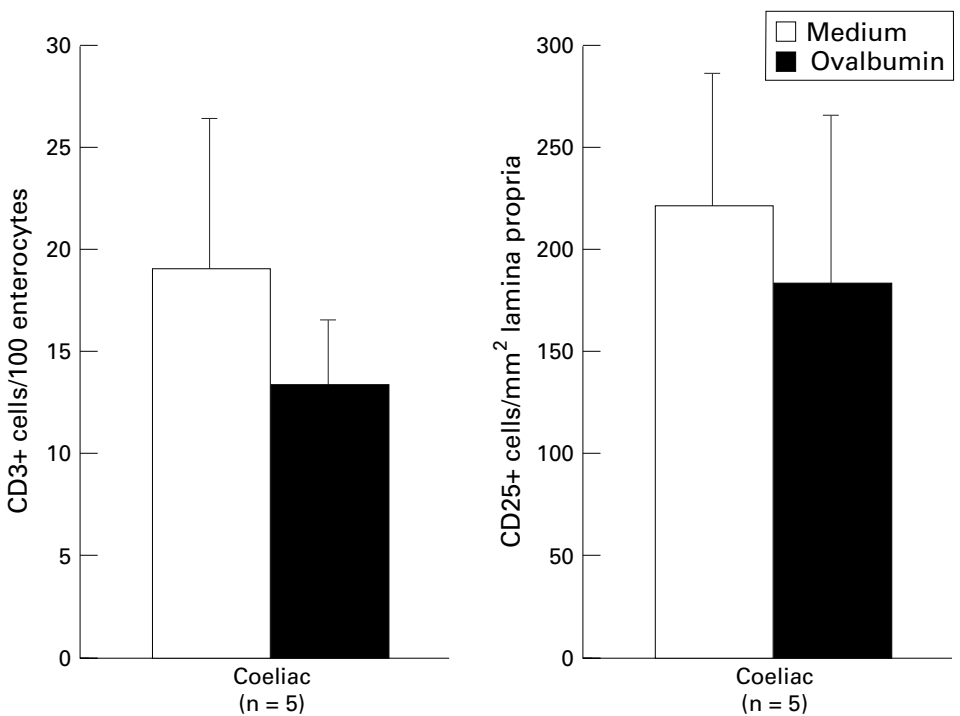

Figure 6 CD3 intraepithelial lymphocytes in rectal biopsy specimens from coeliac patients cultured in vitro with ovalbumin. Bars represent means (SD) of paired samples cultured with medium alone and with ovalbumin. gliadin, as compared with those cultured with medium alone (fig 4). Such an increase was not observed in samples from control subjects (9.3 (5.2) in the presence of gliadin $v 9.3$ (5.1) in the presence of medium alone). In contrast with CD25 cells, no differences were noted between the values observed before (12.8 (6.0)) and after (12.2 (7.3)) culture with medium alone.

A discriminant analysis taking into account the immunohistochemical data was then performed: table 2 lists discriminant function parameters. All controls and all coeliac patients but one were correctly classified (fig 5).

\section{SPECIFICITY OF THE IN VITRO RECTAL RESPONSE}

TO GLIADIN

The in vitro response of rectal mucosa to gliadin was specific for coeliac disease, but also specific for gliadin. In fact, no significant differences were noted between rectal biopsy specimens from coeliac patients cultured with ovalbumin and those cultured with medium only when the number of CD25 cells and intraepithelial CD3 cells were counted (fig 6).

ORGAN CULTURE OF RECTAL MUCOSA FROM SIBLINGS OF COELIAC PATIENTS IN THE PRESENCE OF GLIADIN

In siblings of coeliac patients, lamina propria CD25 cells and intraepithelial CD3 lymphocytes counted in fragments cultured with gliadin (329 (147) and 18.7 (10.4) respectively) were not significantly different when compared with fragments cultured with medium only (275 (108) and 11.1 (5.4) respectively). However, six cases showed a high relative increment ( $>1 \mathrm{SD}$ of the control group) in intraepithelial CD3 cells and three cases a high relative increment in lamina propria CD25 cells. The discriminant score was then calculated for each of the siblings, and the latter three cases were allocated to the coeliac group (fig 5).

The same group of nine siblings had also undergone an in vivo rectal challenge. Intraepithelial and lamina propria CD3 cells were counted six hours after local instillation of gliadin. High percentage increments $(>1 \mathrm{SD}$ of the control group) in intraepithelial CD3 cells were observed in four cases and increments in lamina propria CD 3 cells in two cases. All three subjects allocated to the coeliac group on the basis of in vitro challenge results also showed epithelial infiltration after in vivo challenge. The same subjects underwent jejunal biopsy; in all cases the jejunal architecture was normal, but in two cases an infiltrative pattern was evident, with increased density of intraepithelial CD3 (87 and 61 cells/mm epithelium) and/or $\gamma \delta$ cells (1.3 and 9.6 cells $/ \mathrm{mm}$ epithelium respectively). As far as their HLA haplotype is concerned, one of them showed the HLA DQ heterodimer commonly associated with coeliac disease (HLA DQA $1{ }^{\star} 0501,0201$; $\left.\mathrm{B} 1{ }^{\star} 0201,0201\right)$. HLA DQA $1{ }^{\star} 0201,0201$; $\mathrm{B} 1{ }^{\star} 0201,0201$ and HLA DQA1 0201,0101; $\mathrm{B} 1{ }^{\star} 0201,0503$ were the haplotypes of the two remaining relatives with a positive rectal response. 


\section{Discussion}

Coeliac disease is caused by an immunological process that mainly affects the jejunal mucosa, but the jejunum is not the only site of the gastrointestinal tract involved. In a retrospective review of rectal biopsy specimens, significant inflammatory changes were shown in about a third of such cases. ${ }^{10}$ In more recent years, a series of prospective studies has been conducted by Ensari and colleagues, ${ }^{34}$ which have significantly contributed to the definitive demonstration of gluten dependent inflammatory changes in the rectum of coeliac patients. Their data suggest a continuous $\mathrm{T}$ cell mediated immune response in the rectal mucosa analogous to that in the jejunum. In contrast with the finding in other types of inflammatory colitis or proctitis, the rectal mucosa in coeliac disease lacks any predominance of neutrophils; moreover, computerised image analysis clearly shows that there is no architectural difference between coeliac disease and disease control mucosae. Thus the mechanisms underlying proctitis are probably different from those operating in inflammatory bowel disease, probably depending on the persistence of residual antigenic fragments of gliadin in the colon. Important contributions have come from investigation of the response of rectal mucosa to local gluten challenge. Changes in lamina propria volume and numbers of mast cells, neutrophils, and lymphocytes have been shown in coeliac diseased rectal mucosa after challenge with a peptic-tryptic digest of gluten, ${ }^{5}$ and in a recent study from our laboratory based on the number of epithelial and lamina propria CD3 and $\gamma \delta$ lymphocytes before and six hours after in vivo rectal instillation of gliadin, all coeliac patients were identified by rectal gluten challenge. ${ }^{6}$

Gluten challenge is important not only from a diagnostic point of view, but also beacuse it may provide important information on the early pathological events triggered by the contact with the antigen gliadin. In this respect, in vitro studies are particularly relevant, as they are less invasive. In the present study rectal biopsy samples taken from treated coeliac patients and controls were cultured for 24 hours in the presence of $1 \mathrm{mg} / \mathrm{ml}$ peptic-tryptic digest of gliadin, or in the presence of an unrelated control protein, ovalbumin. In the lamina propria of coeliac biopsy samples cultured in the presence of a peptic-tryptic digest of gliadin, but not in those from controls, the number of cells expressing interleukin 2 receptor were significantly higher than in samples cultured in medium alone; these cells were probably recruited in the subepithelial layer of the lamina propria. No differences were noted in coeliac biopsy specimens cultured with ovalbumin. When adhesion molecules were investigated, expression of VCAM-1 was also enhanced. The latter data parallel in vivo observations, as the rectal mucosa of coeliac patients has shown increased expression of ELAM-1 and VCAM-1, but not ICAM-1, after local gluten challenge. ${ }^{4}$ ELAM-1 has been proposed to be a key endothelial adhesion molecule mediating neutrophil infiltration, and the results of time course experiments ${ }^{4}$ support a major role in neutrophil recruitment. As vascular expression of ELAM-1 in vivo increases significantly by four hours and returns to basal levels by 24 hours, ${ }^{4}$ it is likely that our system based on observations at 24 hours missed such a phenomenon. VCAM-1 expression in vivo had increased eight hours after the challenge, in parallel with an increase in CD3 cells in the lamina propria and epithelium; its expression, analogous to descriptions in experimental models of cell mediated activated mucosal immunity, is the result of $\mathrm{T}$ cell activation. ${ }^{11}$ Another phenomenon observed in rectal biopsy specimens cultured with gliadin is the increased density of intraepithelial CD3 cells. This observation also parallels previous data obtained in vivo, ${ }^{56}$ as well as data obtained from treated small intestinal coeliac mucosa cultured in vitro with gliadin. ${ }^{7}$ In the latter system, gliadin challenge initiates two parallel pathways. One, which precedes $\mathrm{T}$ cell activation, occurs as soon as one hour after gliadin challenge and is characterised by epithelial overexpression of HLA-DR molecules ${ }^{7}$; this phase was not explored in our experiments of rectal mucosa cultures. The second pathway, dominated by $\mathrm{T}$ cell activation, is characterised at 4-12 hours of culture by migration of lamina propria mononuclear cells to the higher mucosal layers, and at 24 hours by expression of T cell activation markers. ${ }^{7}$ These features are also present in the rectal system, although the number of CD25 cells in fragments cultured with medium alone are significantly higher than those noted in the jejunum (206 (88) $v 39$ (10) $/ \mathrm{mm}^{2}$ lamina propria), possibly because of antigenic material passed into the culture medium. Finally, as in the jejunum, in these late steps of culture, there is evidence of intraepithelial lymphocyte migration, a phenomenon that seems not to be a consequence of $\mathrm{T}$ cell activation, as in the jejunal mucosa it is not blocked by the addition of CTLA4-Ig to the culture medium. ${ }^{12}$

In conclusion, our data confirm that, because of its easier access, the rectum may be a useful site for gluten challenge. The cascade of events triggered by contact with the antigen gliadin appears to resemble what happens in the jejunum. It should be noted that the phenomena we have discussed, as explored by in vitro jejunal and rectal challenges, are quite early and occur without mucosal damage and/or an epithelial proliferative response. In other words, in this system it is not possible to explore possible differences between rectum and jejunum in the mechanisms responsible for the severe mucosal damage observed in the small intestine. Nonetheless, this system represents a useful model for studying the mechanisms of activation of the mucosal immune response and may become an essential tool for assessing the antigenic properties of different cereals as well as of different gliadin derived peptides. ${ }^{13}$

This test may prove important also in the investigation of the mucosal immune response to gliadin and for identifying the condition of gluten sensitivity, not only in coeliac patients, 
but, more importantly, in populations predicted to have a high prevalence of abnormal immune response to gliadin. In fact, recent studies have suggested that gluten sensitisation is a condition of abnormal immunity to gluten that is restricted to genetically predisposed individuals, but not exclusive to subjects presenting with a flat mucosa and on a gluten containing diet. In fact, it may be suspected in subjects with smaller changes in mucosal architecture and/or immunological abnormalities such as those that occur in coeliac patients. ${ }^{14}{ }^{15}$ In this regard, first degree relatives of coeliac patients represent a special population. We have recently performed rectal gluten challenge in siblings of coeliac children, ${ }^{10}$ and it was clear that two subgroups were present in such a population, about half of them behaving as coeliacs. The data now provided by in vitro challenge of rectal mucosae with gliadin are consistent with the hypothesis that a significant proportion of first degree relatives are sensitised to gliadin. In vitro rectal gluten challenge, which is relatively less invasive being based on the study of a rectal biopsy fragment, therefore has great potential for defining the epidemiology of gluten sensitisation, particularly in at risk groups, such as first degree relatives of coeliac patients and patients affected by other autoimmune diseases-for example, insulin dependent diabetes.

The expert technical assistance of Mrs Loredana Vacca is gratefully acknowledged. The studies described in this paper were supported by grants from Regione Campania (Protocollo per programmi di diagnosi, cura e riabilitazione delle malattie croniche dell'apparato digerente e della nutrizione dell'infanzia) and Istituto Superiore di Sanita' (Progetto prevenzione dei fattori di rischio nella salute materno-infantile).
1 Marsh MN. Gluten, major histocompatibility complex, and the small intestine. Gastroenterology 1992;102:330-54.

2 Troncone R, Greco L, Auricchio S. Gluten-sensitive enteropathy. Pediatr Clin North Am 1996;43:355-73.

3 Ensari A, Marsh MN, Loft DE, et al. Morphometric analysis of intestinal mucosa. V. Quantitative histological and immunocytochemical studies of rectal mucosae in gluten sensitivity. Gut 1993;34:1225-9.

4 Ensari A, Ager A, Marsh MN, et al. Time-course of adhesion molecule expression in rectal mucosa of glutensensitive subjects after gluten challenge. Clin Exp Immunol sensitive subjects

5 Loft DE, Marsh MN, Sandle GI, et al. Studies of intestinal lymphoid tissue. XII. Epithelial lymphocyte and mucosal responses to rectal challenge in celiac sprue. Gastroenterology 1989;97:29-37.

6 Troncone R, Greco L, Mayer M, et al. In siblings of coeliac children rectal gluten challenge reveals sensitisation to gluten not restricted to coeliac HLA. Gastroenterology 1996; 111:318-24.

7 Maiuri L, Picarelli A, Boirivant M, et al. Definition of the immunological modifications upon in vitro gliadin challenge in the small intestine of coeliac patients. Gastroenterology 1996;110:1368-78.

8 Meeuwisse GW. Diagnostic criteria in coeliac disease. Acta Paediatr Scand 1970;59:461-3.

9 Auricchio S, de Ritis G, De Vincenzi M, et al. Effect of gliadin peptides prepared from hexaploid and tetraploid wheat on cultures of intestine from rat fetuses and coeliac on cultures of intestine from rat fetus

10 Austin L, Dobbins WO. Studies of the rectal mucosa in coeliac sprue: the intraepithelial lymphocyte. Gut $1988 ; 29$; 200-5.

11 Springer TA. Adhesion receptors of the immune system. Nature 1990;346:425

12 Maiuri L, Picarelli A, Coletta S, et al. CTLA4 inhibits T cell mediated gliadin induced immune response in treated coeliac intestinal explants. F Pediatr Gastroenterol Nutr 1996;22: 428.

13 Maiuri L, Troncone $\mathrm{R}$, Mayer $\mathrm{M}$, et al. In vitro activities of A-gliadin related synthetic peptides: damaging effect on the atrophic coeliac mucosa and activation of mucosal immune response in the treated coeliac mucosa. Scand $\mathcal{F}$ Gastroenterol 1996;31:247-53.

14 Holm K, Maki M, Savilahti E, et al. Intraepithelial gamma/ delta $T$ cell receptor lymphocytes and genetic susceptibility to coeliac disease. Lancet 1992;339:1500-3.

15 Holm K, Savilahti E, Koskimies S, et al. Immunohistochemical changes in the jejunum in first degree relatives of patients with coeliac disease and the coeliac marker DQ penes. HL A class II antigen expression, interleukin receptor positive cells and dividing crypt cells. Gut 1994;35:55-60. 